\title{
Interactive Annotation for Event Modality in Modern Standard and Egyptian Arabic Tweets
}

\author{
Rania Al-Sabbagh ${ }^{\dagger}$, Roxana Girju $^{\dagger}$, Jana Diesner $^{\star}$ \\ 'Department of Linguistics and Beckman Institute \\ School of Library and Information Science \\ University of Illinois at Urbana-Champaign, USA \\ \{alsabbal, girju, jdiesner\} Cillinois.edu
}

\begin{abstract}
We present an interactive procedure to annotate a large-scale corpus of Modern Standard and Egyptian Arabic tweets for event modality that comprises obligation, permission, commitment, ability, and volition. The procedure splits up the annotation process into a series of simplified questions, dispenses with the requirement of expert linguistic knowledge, and captures nested modality triggers and their attributes semi-automatically.
\end{abstract}

\section{Introduction}

Event modality, according to Palmer (2001), describes events that are not actualized but are merely potential. It comprises obligation, permission, commitment, ability, and volition. Both obligation and permission emanate from an external authority such as the law; whereas commitments are the obligations placed by speakers on themselves as in promises. Ability is the (in)capacity to do something. Volition is broadly defined as intensions, desires, wishes, and preferences. Event modality is used for several NLP tasks, including sales and marketing analysis (Ramanand et al. 2010, Carlos and Yalamanchi 2012), sentiment analysis (Chardon et al. 2013), the automatic detection of request emails (Lampert et al. 2010), and the classification of animacy and writers' emotions (Liao and Liao 2009, Bowman and Chopra 2012).

To-date, there are no large-scale Arabic corpora annotated for event modality compared to English (Baker et al. 2010, 2012; Rubinstein et al. 2013), Japanese (Matsuyoshi et al. 2010), Portuguese (Hendrickx et al. 2012), and Chinese (Cui and Chi 2013). One obstacle for the creation of modality-annotated corpora is the lack of consensus definitions of modality and its attributes to be rendered into annotation tasks and guidelines. Furthermore, most modality annotation schemes use sophisticated theoretical guidelines that need annotators with linguistic background; hence, annotation typically takes place in in-lab settings at small scales.

In this paper, we present an interactive annotation procedure to annotate event modality and its attributes of sense, polarity, intensification, tense, holders, and scopes in Modern Standard and Egyptian Arabic tweets. The procedure depicts the following ideas: first, it defines each

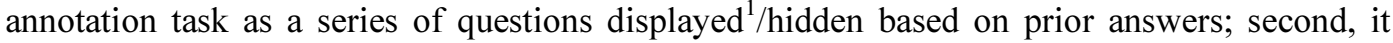
avoids lengthy theoretically-sophisticated definitions and uses the questions instead as simplified self-explanatory annotation prompts; and third, based on the elicited answers it automatically determines nested triggers and their attributes. The fact that our procedure does not require special linguistic background and consists of easy-to-administer questions makes it eligible for large-scale crowdsourcing annotation.

Our corpus comprises 9949 unique tweets, annotated for 12134 tokens that map to 315 unique types of event modality triggers and their attributes of sense, polarity, intensification, tense, holders, and scopes. The reason to work on the genre of tweets is that our corpus is part of a larger project to incorporate linguistic features, such as modality, with network-based features to automatically identify the key players of political discourse on Twitter for countries with fast-changing politics such as Egypt. The fact that our corpus is harvested from the Arabic Egyptian Twitter entails that the corpus is diglossic for Modern Standard Arabic (MSA), the

This work is licensed under a Creative Commons Attribution 4.0 International License. Page numbers and proceedings footer are added by the organizers. License details: http:// creativecommons.org/licenses/by/4.0/ 
formal Arabic variety, and Egyptian Arabic (EA), the native Arabic dialect of Egypt. We evaluate the annotation results with Krippendorff's alpha (Krippendorff 2011). Results show high inter-annotator reliability rates, indicating that our annotation scheme and procedure are effective. The contribution of this paper, therefore, is twofold: first, we create a novel annotated resource for Arabic NLP that is larger than existing corpora even for languages other than Arabic; and second, we present an efficient and easy-to-administer annotation procedure with interactive crowdsourcing potentials.

The rest of this paper is organized as follows: Section 2 outlines the annotation scheme, guidelines and the interactive procedure; Section 3 gives examples for the final output representations; Section 4 describes corpus harvesting and sampling; Section 5 provides the annotation results and disagreement analysis; and Section 6 compares and contrasts our work with related work.

\section{Annotation Scheme: Tasks and Guidelines}

Our annotation scheme comprises six tasks to label sense, polarity, intensification, tense, holders, and scopes for each event modality. Prior to the beginning of the interactive procedure, we highlight all event modalities in each tweet using a string-match algorithm and the lexicons from Al-Sabbagh et al. (2013, 2014a). The algorithm finds all potential event modality triggers (i.e. words/phrases that convey event modality) within each tweet in our corpus and marks them as annotation units. A total of 12134 candidate triggers are highlighted in 9949 tweets.

\subsection{Task 1: Sense}

Sense annotation is to decide for each candidate trigger in context whether it actually conveys event modality given the tweet's context. The same present participle حابب HAbb in example 1 is a volition trigger meaning I want/desire; whereas in example 2 it is a non-modal present participle meaning like/prefer/respect.

1. [مبعا أنا مش حابب [عرو موسى يكسب] TbEA >nA m\$ HAbb [Emrw mwsY yksb]

Definitely, I do not want [Amr Moussa to win].

2. عرو أديب: رسميا الكتاتني مش حابب أبو حامد \#egypt \#qalyoum

Emrw >dyb: rsmyA AlktAtny m\$ $\boldsymbol{H A b \boldsymbol { b }}>$ bw HAmd \#egypt \#qalyoum

Amr Adeeb: Alkatatny does not officially like Abu Hamed \#egypt \#qalyoum

We define sense annotation as a synonymy judgment task, following Al-Sabbagh et al. (2013, 2014b). Each event modality sense is represented by an exemplar set manually selected so that: (1) each exemplar is an unambiguous event modality trigger; (2) exemplars are in both MSA and EA; (3) exemplars comprise both simple words and multiword expressions; (4) exemplars are both affirmative and negative; and (5) exemplars are of different intensities. Presented with a pre-highlighted candidate trigger in context and the exemplar sets, annotations are to decide whether the candidate trigger is synonymous with any of the exemplar sets. If not, the trigger is then assumed as non-modal.

If an annotator decides that a given candidate trigger is a non-modal, no further questions about polarity, intensification, tense, holders, or scopes are displayed. In order to guarantee that annotators do not select the non-modal option as an easy escape, they are not allowed to move forward without giving at least one synonym of their own to the candidate trigger.

\subsection{Task 2: Polarity}

Task 2 uses as input the candidates labeled as valid event modality triggers in Task 1 and label each as either affirmative (AFF) or negative (NEG). To decide, annotators are instructed to consider the absence/presence of:

- Negation particles such as مش غير gyr (not), among others.

- Negation affixes, especially in EA, like the circumfix $m \ldots$... مقدرش $m q d r \$$ (I cannot).

\footnotetext{
${ }^{1}$ Throughout the examples, modality triggers are marked in boldface, and scopes are in-between brackets.
} 
- Negative polarity items like عمري Emry (never) and لم يعد lm yEd (no longer).

- Negative auxiliaries where negation is placed on the past tense auxiliary as in مكنش عايز $m k n t \$ E A y z$ (I did not want).

- Inherently-negative triggers that encode negation in their lexical meanings such as EAjz (incapable) and ين ymnE (prohibit).

- Embedding under negated epistemic modality triggers as in أعتقد أنه يجب la $l A$ أEtqd $>n h$ $y j b$ (I do not think it is necessary) which entails that the speaker is not actually setting an obligation.

Annotators are instructed that using multiple negation markers results in an affirmative sense. Thus, لم المجز lm yEjz (he was not unable to) means that he was actually able to. Annotators are required to give the reason for negation if they decide that a given trigger is negative.

\subsection{Task 3: Intensification}

Event modality triggers have different lexical intensities (i.e. intensities encoded in the lexical meaning of the word/phrase regardless of the context). In obligation triggers, for instance, even without a context, Arabic speakers know that ضروري Drwry (necessary) expresses a higher necessity than المفروض AlmfrwD (should). When used in context, the trigger's lexical intensity can be maintained as is, or amplified/mitigated by such linguistic means as:

- Modification: adverbs like تُ $\operatorname{tmAmA}$ (absolutely) amplify lexical intensity; whereas mitigation is invoked by such adverbs as غالبا gAlbA (most probably).

- Categorical negation typically amplifies lexical intensity as in مش المفروض أبدا Almfrw $D>b d A$ (it should never be).

- Emphatic expressions such as و واله $q d$ (indeed) (I swear), and من كل قلبي $m n k l$ qlby (wholeheartedly), among others, lead to lexical intensity amplification.

- Coordination of two or more triggers typically results in intensity amplification as in lAzm wDrwry (must and necessary).

- Embedding under epistemic modality triggers can affect the lexical intensities of event modality triggers. In أعتقد من الضروري أن >Etqd mn AlDrwry >n (I think it is necessary to) the strong obligation associated with الضروري AlDrwry (necessary) is mitigated by the moderate-intensity epistemic أعتى >Etqd (I think), being embedded under it.

The annotators' task for intensification annotation is to decide for each candidate labeled as a valid event modality trigger in Task 1 whether its lexical intensity is amplified (AMP), mitigated (MTG) or maintained (AS IS). During interactive annotation, annotators are asked to provide the reason for their selection; that is, whether the lexical intensity is affected by modification, coordination, negation, embedding or any other reason whether listed above or not.

\subsection{Task 4: Tense}

In this version of our event modality corpus, we work on the present and past tenses only. Thus, Task 4 is to decide for each valid event modality trigger from Task 1 whether it is present (PRS) or past (PST). Annotators are required to give their reasons for selecting either PRS or PST.

\subsection{Task 5: Holders}

Holder annotation identifies the source of the obligation, permission, commitment, ability, or volition. In example 3, the source that sets the obligation that Egyptians have to learn the meaning of democracy is the Twitter user.

3. لازم [المصريين يتعلموا يعني إيه ديموقر اطية الأول]

IAzm [AlmSryyn ytElmwA yEny <yh dymwqrATyp Al>wl]

[Egyptians have to learn what democracy is first]

The holder is not always the Twitter user, however. In example 4, the Twitter user quotes Kamal Alganzoury - a former Egyptian Prime Minster - stating that he does not want to 
لبس لدي رغبة continue as the Prime Minister. Therefore, the holder of the negated volition trigger lys ldy rgbp (not have a will) is Alganzoury not the Twitter user. This is an example of the nested holder notion first proposed by Wiebe et al. (2005) and Saurí and Pustejovsky (2009).

4. الدكتور كمال الجنزوري: لليس لاي رغبة في [الاستمر ار ] HSCAF \#Tahrir \#Egypt Aldktwr kmAl Aljnzwry: lys ldy rgbp fy [AlAstmrAr] \#SCAF \#Tahrir \#Egypt Dr. Kamal Alganzoury: I do not wish to [continue] \#SCAF \#Tahrir \#Egypt

Another example of nested holders is example 5. We know that the regime is incapable of maintaining security and protecting the people only because the Twitter user says so. Put differently, the best way to understand this tweet is that according to what the Twitter user holds as a true proposition, the regime is unable to maintain security and protect the people.

5. النظام غير قادر على [توفير الأمن أو حماية المو اطنين] AlnZAm gyr qAdr ElY [twfyr Al>mn >w HmAyp AlmwATnyn]

The regime is not able to [maintain security and protect the people]

We can have two or more nested holders. In example 4, the two holders are Alganzoury who expresses his unwillingness to continue as a Prime Minster and the Twitter user who is quoting Alganzoury. In example 5, the two holders are the regime that is incapable of marinating security and protecting its people and the Twitter user who holds this proposition as true. In example 6, we have three nested holders: the Iranians who are unwilling to confront the outside world, Obama who holds that as a true proposition about Iranians, and the Twitter user who is quoting Obama stating his proposition.

6. اوباما: الثنعب الاير اني لم يعد يرغب في [المواجهة مع العالم الخارجي] AwbAmA: Al\$Eb AlAyrAny Im yEd yrgb fy [AlmwAjhp mE AlEAlm AlxArjy]

Obama: the Iranians no longer want to [confront the other countries].

During the interactive procedure, annotators are first asked whether the holder is the same as the Twitter user. If not, more questions are displayed to determine (1) who the real holder is; (2) whether the tweet is a(n) (in)direct quote; or it conveys the Twitter user's assumptions.

When the holder is not the Twitter user, annotators are asked to mark the boundaries of the linguistic unit that corresponds to the holder in the tweet's text. Annotators are instructed to use the maximal length principle from Szarvas et al. (2008) so that they mark the largest possible meaningful linguistic unit. Thus, in example 4 the holder is الدكتور كمال الجنزوري Aldktwr kmAl Aljnzwry (Dr. Kamal Alganzoury) not only Kamal Alganzoury.

\subsection{Task 6: Scopes}

Scopes are the events modified by the trigger, syntactically realized as clauses, verb phrases, deverbal nouns or to-infinitives, according to Al-Sabbagh et al. (2013). We use the same maximal length principle from Task 5 so that the marked scope segment corresponds to the largest meaningful linguistic unit that describes the event. Typically, scope segments are delimited by: (1) punctuation markers and (2) subordinate conjunctions.

Annotators are instructed that: (1) a single trigger may have one or more scopes; (2) two or more triggers - especially conjoined by coordinating particles - can share the same scope; and (3) scopes are not necessarily adjacent to their triggers. Examples 7, 8 and 9 illustrate each of these guidelines, repecetively.

7. لو استبعد شفيق يستطيع [الطعن] و[العودة لسباق الرئاسة] lw AstbEd \$fyq ystTyE [AlTEn] w[AlEwdp lsbAq Alr\} Asp]

If Shafiq is excluded, he can [appeal] and [run again for presidency].

8. mlAyyn AlmSryyn Ally brh mSr lAzm wHtmA wDrwry wyjb [ybqY lhm Hq AltSwyt]

It is necessary, it is a must, it is a need that [Egyptians abroad are given the right to vote].

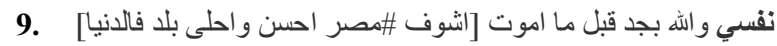
nfsy wAllh bjd qbl mA Amwt [A \$wf \#mSr AHsn wAHlY bld fAldnyA]

I really wish before I die to [see \#Egypt becoming one of the best counties in the world].

\section{Final Output Representation}

All elicited answers during annotation are organized into the representations illustrated in the following examples. The representation of example 10 reads as: the Twitter USER strongly did 
not want Shafiq to win the presidential elections. The trigger اتمنيت Atmnyt (wished) is tagged as synonymous with the volition exemplar set; therefore, it denotes a DESIRE. It is then labeled as a past tense (PST), negative (NEG) trigger. Furthermore, its lexical intensity is labeled as amplified (AMP) because of the categorical negation عمري ما Emry $m A$ (never ever). Originally, تمنيت Atmnyt (wished) is of moderate lexical intensity, being less intense than اشتهيت A\$thyt (longed for) but more intense than أردت >rdt (wanted). Given the categorical negation, the lexical intensity of اتمنيت Atmnyt (wished) goes up the scale from moderate to strong (STRG).

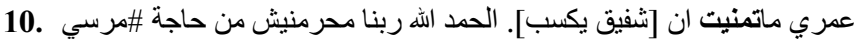

Emry mAtmnyt An [\$fyq yksb]. AlHmd Allh rbnA mHrmny\$ mn HAjp \#mrsy

I have never ever wished [Shafiq to win]. Thank God! \#Morsi.

rep. USER, STRG PST NEG DESIRE ( $\$ f y q y k s b)$

Example 11 reads as: the Twitter USER reports Hegazy stating that he has the ability to become the Muslim's caliphate. The trigger أصلح >SlH (can) is labeled as synonymous with the ability exemplar set. It is also labeled as a present (PRS), affirmative (AFF) trigger whose lexical intensity is maintained (AS IS) in the context. Therefore, its lexical intensity is maintained to its original level which is moderate (MOD).

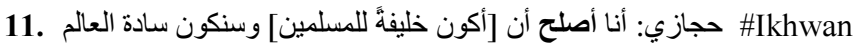
HjAzy: >nA >SIH >n [> kwn xlyfp llmslmyn] wsnkwn sAdp AlEAlm \#Ikhwan Hegazy: I can [be the Muslims' caliphate] and we will become the world's masters. \#Ikhwan rep. USER, report, (HjAzy, MOD PRS AFF ABLE, (> kwn xlyfp llmslmyn))

Example 12 shows a Twitter user who holds as true that the only thing Egypt needed was a wise politician to avoid the bloodshed. The trigger تحتاج tHtAj (needs) is labeled as an obligation trigger synonymous with تنطلب ttTlb (requires). It is also labeled as past tense (PST) given the preceding past tense auxiliary itkn (was). The assigned strong (STRG) lexical intensity label is attributed to the fact that the original moderate intensity of تحتاج $t H t A j$ (needs) is amplified by

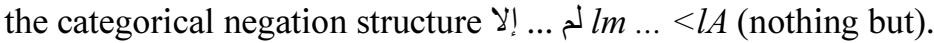

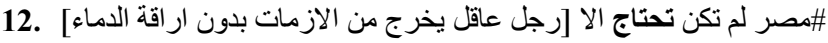
\#mSr Im tkn tHtAj AlA [rjl EAql yxrj mn AlAzmAt bdwn ArAqp AldmA']

\#Egypt needed nothing but [a rational politician who solves crises without bloodshed] rep. USER, true, ( $m S r$, STRG PST AFF REQUIRE ( $r j l$ EAql yxrj mn AlAzmAt bdwn ArAqp AldmA'))

Example 13 illustrates the representation of three-level nested holders. It reads as: the USER reports Obama's assumption as the latter holds as true that the Iranians do not want to confront other countries.

13. اوباما: الثعب الاير اني لم يعد يرغب في [المواجهة مع العالم الخارجي]

AwbAmA: Al\$Eb AlAyrAny Im yEd yrgb fy [AlmwAjhp mE AlEAlm AlxArjy]

Obama: the Iranians no longer want to [confront other countries].

rep. USER, report, (AwbAmA, true, (Al\$Eb AlAyrAny, MOD PRS NEG DESIRE, (AlmwAjhp mE AlEAlm AlxArjy)))

Example 14 shows how two conjoined triggers (i.e. لازم IAzm (must) and Drwry (necessary)) that share the same holder and scope are merged into one representation, and the conjunction leads to amplifying the intensity of the obligation set by them both.

14. لازم وضروري [كلنا نكون قدام مقر المحاكمة ومعانا صورة الرئيس]

IAzm wDrwry [klnA nkwn qdAm mqr AlmHAkmp wmEAnA Swrp Alr\}ys]

We must and it is necessary that [we go to the court with President's pictures].

rep. USER, STRG PRS AFF REQUIRE, (klnA nkwn qdAm mqr AlmHAkmp wmEAnA Swrp Alr\}ys)

\section{Corpus Harvesting}

Tweets are harvested from the Arabic Egyptian Twitter provided that (1) each tweet has at least one trendy political English or Arabic hashtag; and (2) each tweet has at least one candidate event modality trigger from the Arabic modality lexicons (Al-Sabbagh et al. 2013, 2014a). We harvest tweets from a variety of users such as newspapers, TV stations, political and humanitarian campaigns, politicians, celebrities, and ordinary people. Thus, our corpus comprises both MSA, the formal Arabic variety, and EA, the native Arabic dialect of Egypt. The harvested corpus comprises 9949 unique tweets, with 12134 tokens of event modality triggers that map to 315 unique types. 


\section{Annotation Results}

\subsection{Evaluation Methodology and Metrics}

Our annotation tasks are of two types: (1) Tasks 1-4 are label-based where there is a pre-defined set of labels from which annotators choose; and (2) Tasks 5-6 are segmentation-based where the output of the annotation is a text segment. For the segmentation-based tasks, we use an all-ornothing method to measure inter-annotator reliability: for segments to be considered as agreement, they must share both the beginning and end boundaries. We use Krippendorff's alpha $\alpha$ (Krippendorff 2011) as our inter-annotator reliability measure, following the most recent work on modality annotation for other languages including English (Rubinstein et al. 2013) and Chinese (Cui and Chi 2013). For more details on Krippendorff's alpha and a, we refer the reader to Artstein and Poesio (2008).

\subsection{Results}

We use the surveygizmo survey services ${ }^{2}$ to implement our interactive annotation procedure given that their survey structure is one that uses conditional branching and skip logic. We distribute the survey on Twitter and we have three annotators participating. According to the short qualifying quiz given at the beginning of the survey, all three participants are native Egyptian Arabic (EA) speakers who have at least two-year experience with Twitter. They are also university graduates who, therefore, master MSA. None of the participants has a linguistics background. Table 1 shows alpha rates for each annotation task.

\begin{tabular}{lcccccc} 
& Sense & Polarity & Intensification & Tense & Holder & Scope \\
\hline Obligation & 0.890 & 0.893 & 0.892 & 0.978 & 0.829 & 0.744 \\
Permission & 0.864 & 0.905 & 0.821 & 0.983 & 0.800 & 0.739 \\
Commitment & 0.760 & 0.794 & 0.783 & 0.947 & 0.702 & 0.654 \\
Ability & 0.895 & 0.914 & 0.905 & 0.950 & 0.828 & 0.763 \\
Volition & 0.921 & 0.921 & 0.867 & 0.982 & 0.858 & 0.779 \\
\hline Averages & $\mathbf{0 . 8 6 6}$ & $\mathbf{0 . 8 8 5}$ & $\mathbf{0 . 8 5 4}$ & $\mathbf{0 . 9 6 8}$ & $\mathbf{0 . 8 0 3}$ & $\mathbf{0 . 7 3 6}$ \\
\hline
\end{tabular}

Table 1: Krippendorff's alpha rates for inter-annotator reliability

\subsection{Discussion and Disagreement Analysis}

Among the factors that lead to high inter-annotator reliability are that: (1) the vast majority of negation is explicitly marked by negation particles that are easy to detect by human annotators; (2) the vast majority of triggers are used without any amplification or mitigation markers; and (3) punctuation markers are surprisingly informative for marking scope boundaries and direct quotations; and hence, holders.

Sense-related disagreement is attributed to: (1) nominal triggers, (2) highly-polysemous triggers, and (3) different interpretations invoked by the -RATIONAL (i.e. non-human) holders.

Typically, event modality triggers are adjunct constituents that add an extra-layer of meaning and can be removed without disturbing the syntactic structure. Yet, in example 15, واجب w Ajb (a must) and أوجب >wjb (a more important must) have main grammatical functions as the predicates of the phrases they modify. Most of the exemplars from Section 2.1 are adjuncts; and, thus, none can substitute و و اجب >wjb (a more important must) in such a context.

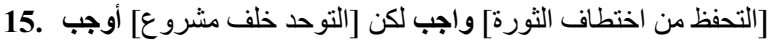

[AltHfZ mn AxtTAf Alvwrp] wAjb lkn [AltwHd xlf $m \$ r w E]>\boldsymbol{w j} \boldsymbol{b}$

[Being cautious about manipulating the revolution] is a must but [getting united for one project] is a more important must.

Highly-polysemous triggers invoke disagreement because in many cases even the context is ambiguous. In example 16, أقسم >qsm (I swear) has two eligible interpretations: an epistemic trigger interpretation I assure (you) that and a commitment trigger interpretation I promise (you)

\footnotetext{
${ }^{2}$ http://www.surveygizmo.com/
} 
that. Even the context is not enough to disambiguate the two interpretations and annotators go by the most common sense for the trigger according to their own opinions.

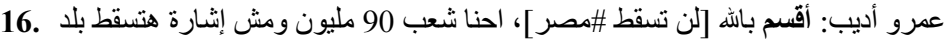

Emrw >dyb: >qsm bAllh [ln tsqT \#mSr], AHnA \$Eb 90 mlywn wm\$<\$Arp htsqT bld

Amr Adeeb: I promise/assure (you) by God that [\#Egypt will not collapse]. We are 90 million Egyptians and we will not be defeated by a sign.

Non-human or -RATIONAL holders invoke disagreement, especially for obligation versus volition triggers. The most common sense of such triggers as EAyzp (want) is volition. Yet, when the holder is -RATIONAL like الانتخابات AlAntxAbAt (the elections) in example 17, annotators disagree as to whether عAyzp means want (i.e. a volition trigger) or need (i.e. an obligation trigger).

17. الانتخابات عايزة [مرشحين] وحملات الأحز اب تيجي بر احتها

AlAntxAbAt EAyzp [mr\$Hyn] wHmlAt Al>HzAb tyjy brAHthA

Elections want/need [candidates] and later we can establish the political parties.

Intensity-related disagreement is attributed mostly to progressive verb aspect. Some annotators consider progressive verb aspect as indicated by the EA prefix $b$ as a marker for lexical intensity amplification. Thus they tag the volition trigger بtmn $Y$ (I wish) in example 18 as amplified, especially it is modified by كل يوم $k l$ ywm (everyday).

18. كل يوم بتمنى [سقوط حكم

kl ywm btmn Y [sqwT Hkm \#mrsy]

Every day, I wish for [\#Morsi's regime to fall].

Polarity-related disagreement is mainly caused by (1) negated holders and (2) contextual negation. In مفيش حد يقدر mfy \$ Hd yqdr (no one can), annotators disagree as to whether يقدر yqdr (can) should be labeled as affirmative or negative. By contextual negation we mean examples like من الصعب أن نتمنى أن AlSEb >n ntmnY >n (it is hard to wish to), which entails negation due to the adjective الصعب i AlSEb (hard).

Holder-related disagreement is attributed mainly to generic nouns and impersonal pronouns like الواحد Al\$E AlwAHd (one), respectively. They are interpreted by some annotators as referring implicitly to the Twitter USER. Therefore, the annotators select the USER as the only holder with zero nesting. Other annotators interpret them as referring to people in general not necessarily the Twitter USER and thus they consider these as instances of nested holders.

Scope-related disagreement is attributed to (1) ambiguous subordinate conjunctions, (2) triggers' modifiers, and (3) absent punctuation markers.

Tense yields almost perfect inter-annotator reliability rates. Annotation disagreement does not show any particular pattern. Therefore, we attribute minor disagreement to random errors, resulting from fatigue.

\subsection{Majority Statistics}

Based on majority annotations, Table 2 gives the statistics for our corpus in terms of sense, polarity, intensification, and tense. As for holder annotations, approximately $60.5 \%$ of the triggers have zero-nested holders (i.e. the tweet's writer is the same as the holder).

\begin{tabular}{l|ll|ll|lll|ll}
\cline { 2 - 9 } & \multicolumn{2}{c|}{ Sense } & \multicolumn{2}{c|}{ Polarity } & \multicolumn{3}{c|}{ Intensification } & \multicolumn{2}{c}{ Tense } \\
\cline { 2 - 10 } & MD & NMD & AFF & NEG & AMP & MTG & ASIS & PRS & PST \\
\hline Ability & 1729 & 920 & 1047 & 682 & 348 & 308 & 1073 & 1175 & 554 \\
Commitment & 1048 & 495 & 599 & 449 & 221 & 220 & 607 & 639 & 409 \\
Obligation & 1786 & 848 & 1059 & 727 & 369 & 399 & 1018 & 1018 & 768 \\
Permission & 1699 & 980 & 1054 & 645 & 286 & 428 & 985 & 1053 & 646 \\
Volition & 1622 & 1007 & 974 & 648 & 341 & 292 & 989 & 1038 & 584 \\
\hline \multicolumn{1}{c}{ Totals } & $\mathbf{7 8 8 4}$ & $\mathbf{4 2 5 0}$ & $\mathbf{4 7 3 3}$ & $\mathbf{3 1 5 1}$ & $\mathbf{1 5 6 5}$ & $\mathbf{1 6 4 7}$ & $\mathbf{4 6 7 2}$ & $\mathbf{4 9 2 3}$ & $\mathbf{2 9 6 1}$
\end{tabular}

Table 2: Token statistics for each annotation task per event modality sense where MD is modal, NMD is non-modal, AFF is affirmative, NEG is negative, AMP is amplified, MTG is mitigated, ASIS is as is, PRS is present, and PST is past 


\section{Related Work}

Event modality is the focus of many annotation projects. Matsuyoshi et al. (2010) annotate a corpus of English and Japanese blog posts for a number of modality senses including volition, wishes, and permission. They annotate sense, tense, polarity, holders as well as other attributes that we have not covered in our scheme such as grammatical mood. They report macro kappa inter-annotator agreement rates of $0.69,0.70,0.66$ and 0.72 for holders, tense, sense, and polarity, respectively.

Baker et al. $(2010,2012)$ simultaneously annotate modality and modality-based negation for Urdu-English machine translation systems. Among the modality senses they work on are requirement, permission, success, intention, ability, and desires. They report macro kappa interannotator agreement rates of 0.82 for sense annotation and 0.76 for scopes. They, however, do not annotate holders and do not consider nested modalities.

Hendrickx et al. (2012) annotate eleven modality senses in Portuguese, including necessity, capacity, permission, obligation, and volition, among others. They report a macro kappa interannotator rate of 0.85 for sense annotation.

Rubinstein et al. (2013) propose a linguistically-motivated annotation scheme for modalities in the MPQA English corpus. They annotate sense, polarity, holders, and scopes, among other annotation units. They work on obligation, ability, and volition among other modality senses. They attain macro alpha inter-annotator reliability rates of 0.89 and 0.65 for sense and scope, respectively.

Cui and Chi (2013) apply the same scheme of Rubinstein et al. (2013) to the Chinese Penn Treebank and get alpha inter-annotator reliability rates of 0.81 and 0.39 for sense and scope annotation, respectively.

Finally, Al-Sabbagh et al. (2013) annotate event modality in MSA and EA tweets. We attain kappa inter-annotator agreement rates of 0.90 and 0.93 for sense and scope annotation, respectively, for only 772 tokens of event modality triggers.

Our annotation results, therefore, are comparable to the results in the literature. Furthermore, our annotation scheme and its tasks are orthogonal to most of the aforementioned schemes. However, the key differences between our work and related work are:

- We use a standardized taxonomy of event modality - Palmer's (2001) - that has been proved valid for a variety of languages, including Arabic, according to Mitchell and Al-Hassan (1994), Brustad (2000), and Moshref (2012).

- We annotate nested holders unlike some of the aforementioned studies (e.g. Baker et al. 2010,2012) and use a wider range of negation and intensification markers.

- We use crowdsourcing with simplified guidelines implemented interactively to annotate a larger-scale corpus of 12134 tokens for event modality and its attributes.

\section{Conclusion and Outlook}

We presented a large-scale corpus annotated for event modality in MSA and EA tweets. We use a simplified annotation procedure that defines each annotation task as a series of questions, implemented interactively. Our scheme covers a wide range of the most common annotation units mentioned in the literature, including modality sense, polarity, intensification, tense, holders, and scopes. We deal with nested holders - which are crucial in a highly interactive genre such as tweets where users frequently quote others and make assumptions about them. We also automatically merge triggers with shared holders and scopes based on elicited annotators' answers. The annotation procedure yields reliable results and creates a novel resources for Arabic NLP. The current version of our corpus does not, however, cover a number of issues including: the future tense, grammatical moods other than the declarative, and modality entailment. By modality entailment, we mean, for example, when a tweet's user criticizes the obligation of another quoted person, this entails that the user does not consider such an event as required. For a future version of the corpus, we plan to cover such points. Furthermore, we will use the corpus to train and test a machine learning system for the automatic processing of Arabic event modality. 


\section{References}

Rania Al-Sabbagh, Jana Diesner and Roxana Girju. 2013. Using the Semantic-Syntactic Interface for Reliable Arabic Modality Annotation. In Proceedings of IJCNLP'13, pages 410-418, October 14-18, 2013, Nagoya, Japan.

Rania Al-Sabbagh, Roxana Girju and Jana Diesner. 2014a. Unsupervised Construction of a Lexicon and a Pattern Repository of Arabic Modal Multiword Expressions. In Proceedings of the $10^{\text {th }}$ Workshop of Multiword Expressions at EACL'14, April 26-27, 2014, Gothenburg, Sweden.

Rania Al-Sabbagh, Roxana Girju and Jana Diesner. 2014b. 3arif: A Corpus of Modern Standard and Egyptian Arabic Tweets Annotated for Epistemic Modality Using Interactive Crowdsourcing. In Proceedings of the $25^{\text {th }}$ International Conference on Computational Linguistics, August 23-29, 2014, Dublin, Ireland.

Ron Artstein and Massimo Poesio. 2008. Inter-Coder Agreement for Computational Linguistics. Computational Linguistics, volume 34, issue 4, pages 555-596.

Kathrin Baker, Michael Bloodgood, Mona Diab, Bonnie Dorr, Nathaniel W. Filardo, Lori Levin and Christine Piatko. 2010. A Modality Lexicon and its Use in Automatic Tagging. In Proceedings of the $7^{\text {th }}$ International Conference on Language Resources and Evaluation (LREC'10), pages 1402-1405, May 19-21, 2010, Valetta, Malta.

Kathryn Baker, Michael Bloodgood, Bonnie J. Dorr, Chris Callison-Burch, Nathaniel W. Filardo, Christine Piatko, Lori Levin and Scott Miller. 2012. Modality and Negation in SIMT. Computational Linguistics. volume 38, issue 2, pages 411-438.

Samuel R. Bowman and Harshit Chopra. 2012. Automatic Animacy Classification. In Proceedings of the NAACL HTL 2012 Student Research Workshop, pages 7-10, June 3-8, 2012, Montreal, Canada.

Kristen E. Brustad. 2000. The Syntax of Spoken Arabic: A Comparative Study of Moroccan, Egyptian, Syrian and Kuwaiti Dialects. Georgetown University Press, Washington DC, USA.

Cohan Sujay Carlos and Madulika Yalamanchi. 2012. Intention Analysis for Sales, Marketing and Customer Service. In Processing of COLING 2012: Demonstration Papers, pages 33-40, December 2012, Mumbai, India.

Baptiste Chardon, Farah Benamara, Yannick Mathieu, Vladimir Popescu and Nicholas Asher. 2013. Sentiment Composition Using a Parabolic Model. In Proceedings of the $10^{\text {th }}$ International Conference on Computational Semantics (IWCS 2013), pages 47-58, March 20-22, 2013, Potsdam, Germany.

Yanyan Cui and Ting Chi. 2013. Annotating Modal Expressions in the Chinese Treebank. In Proceedings of the IWC 2013Workshop on Annotation of Modal Meaning in Natural Language (WAMM), pages 2432, March 2013, Potsdam, Germany.

Iris Hendrickx, Amàlia Mendes and Silvia Mencarelti. 2012. Modality in Text: A Proposal for Corpus Annotation. In Proceedings of the $8^{\text {th }}$ International Conference on Language Resources and Evaluation (LREC'12), pages 1805-1812, May 21-27, 2012, Istanbul, Turkey.

Klaus Krippendorff. 2011. Computing Krippendorff's Alpha-Reliability. Annenberg School of Communication, Departmental Papers: University of Pennsylvania.

Andrew Lampert, Robert Dale and Cecile Paris. 2010. Detecting Emails Containing Requests for Action. In Proceedings of Human Language Technologies: the 2010 Annual Conference of the North American Chapter of the ACL, pages 984-992, June 2010, Los Angles, California.

Ying-Shu Liao and Ting-Gen Liao. 2009. Modal Verbs for the Advice Move in Advice Columns. In Proceedings of the $23^{\text {rd }}$ Pacific Asia Conference on language, Information and Computation, pages 307-316, December 3-5, 2009, Hong Kong, China.

Suguru Matsuyoshi, Megumi Eguchi, Chitose Sao, Koji Murakami, Kentaro Inui and Yuji Matsumoto. 2010. Annotating Event Mentions in Text with Modality, Focus and Source Information. In Proceedings of LREC'10, pages 1456-1463, May 19-21, 2010, Valletta, Malta.

T. F. Mitchell and S. A. Al-Hassan. 1994. Modality, Mood and Aspect in Spoken Arabic with Special Reference to Egypt and the Levant. London and NY: Kegan Paul International. 
Ola Moshref. 2012. Corpus Study of Tense, Aspect, and Modality in Diglossic Speech in Cairene Arabic. PhD Thesis. University of Illinois at Urbana-Champaign.

Frank R. Palmer. 2001. Mood and Modality. $2^{\text {nd }}$ Edition. Cambridge University Press, Cambridge, UK.

J. Ramanand, Krishna Bhavsar and Niranjan Pedanekar. 2010. Wishful Thinking: Finding Suggestions and "Buy" Wishes for Product Reviews. In Proceedings of the NAACL HLT 2010 Workshop on Computational Approaches to the Analysis and Generation of Emotion in Text, pages 54-61, June 2010, Los Angeles, California.

Aynat Rubinstein, Hillary Harner, Elizabeth Krawczyk, Daniel Simoson, Graham Katz and Paul Portner. 2013. Toward Fine-Grained Annotation of Modality in Text. In Proceedings of the IWC 2013Workshop on Annotation of Modal Meaning in Natural Language (WAMM), pages 38-46, March 2013, Potsdam, Germany.

Roser Saurí and James Pustejovsky. 2009. FactBank: A Corpus Annotated with Event Factuality. Language Resources and Evaluation, 43:227-268

György Szarvas, Veronika Vincze, Richárd Farkas and János Csirik. 2008. The BioScope Corpus: Annotation for Negation, Uncertainty and their Scope in Biomedical Texts. In Proceedings of BioNLP 2008: Current Trends in Biomedical Natural Language Processing, pages 38-45, June 2008, Columbus, Ohio, USA.

Janyce Wiebe, Theresa Wilson and Claire Cardie. 2005. Annotating Expressions of Opinions and Emotions in Language. Language Resources and Evaluation, volume 39, issue 203, pages 1663-210. 